\title{
Approximate weak amenability, derivations and Arens regularity of Segal algebras
}

\author{
by \\ Fereidoun Ghahramani (Winnipeg) and \\ Anthony To-Ming LaU (Edmonton)
}

\begin{abstract}
We continue our study of derivations, multipliers, weak amenability and Arens regularity of Segal algebras on locally compact groups. We also answer two questions on Arens regularity of the Lebesgue-Fourier algebra left open in our earlier work.
\end{abstract}

0. Introduction. In [9] we studied derivations, weak amenability and Arens regularity of certain classes of Segal algebras on locally compact groups and in particular we showed that every symmetric Segal algebra $S(G)$ on an amenable SIN group is approximately weakly amenable, i.e., for every continuous derivation $D: S(G) \rightarrow S(G)^{*}$, there is a net $\left(\varphi_{i}\right) \subset S(G)^{*}$ such that

$$
D(f)=\lim _{i \rightarrow \infty}\left[f \cdot \varphi_{i}-\varphi_{i} \cdot f\right] \quad(f \in S(G)) .
$$

In this paper we continue our study of derivations, multipliers, weak amenability and Arens regularity of Segal algebras and in particular we show that a symmetric Segal algebra $S(G)$ is approximately weakly amenable if one only assumes that $G$ is a SIN group or an amenable group. We show that if $G$ is amenable and $S(G)$ is a symmetric Segal algebra, then for every Banach $L^{1}(G)$-bimodule $X$, continuous derivations from $S(G)$ into $X$ are approximately inner. Alternatively, continuous derivations from $S(G)$ into $X^{*}$ are precisely the ones defined by continuous double centralizers.

In [9] we also showed that the Lebesgue-Fourier algebra of a compact (discrete) group $G$ is Arens regular for the convolution (resp. pointwise) product. Here we prove the converse to these results, provided that $G$ is unimodular in the first case, and for all groups in the second case. Thus we answer two questions left open in [9].

For a compact group $G$, we determine the space $\mathcal{Z}^{1}(S(G), X)$ of continuous derivations from $S(G)$ into $X$, where $S(G)$ is one of the naturally arising

2000 Mathematics Subject Classification: 43A20, 43A22, 46A20, 46H25.

Research of the first named author supported by NSERC Grant 36640-02.

Research of the second named author supported by NSERC Grant A-7679. 
Segal algebras $(C(G), *),\left(L^{p}(G), *\right), 1 \leq p \leq \infty$, or $(A(G), *)$ where $*$ stands for the convolution product and $X$ is a naturally arising $S(G)$-module. Along the way we determine all the left (right) multipliers from $S(G)$ into $X$. Finally, we find necessary conditions for $S(G)$ to be Arens regular; these conditions are sometimes sufficient as well. We also find necessary and sufficient conditions for $S(G)$ to be an ideal in its second dual space, when the latter is equipped with the first or the second Arens product.

Our interest in (co)homological properties of Segal algebras stemmed from our study of the Lebesgue-Fourier algebra $\mathcal{L} A(G)$ (see Preliminaries for definition and [9] for further properties) at a time when it was proved by H. G. Dales and S. S. Pandey that certain Segal algebras on abelian locally compact groups are weakly amenable [4]. As pointed out in [4] a proper Segal algebra can never be amenable, since it cannot have a bounded approximate identity. However it is our opinion that Segal algebras provide good examples for generalized notions of amenability that are recently being developed. For example, it has been shown by the first named author and R. J. Loy that every symmetric Segal algebra on an amenable group is essentially amenable ([10, Corollary 7.1]).

We thank the referee for his/her many valuable suggestions.

1. Preliminaries. Throughout this paper, $G$ will denote a locally compact group with a fixed left Haar measure. Given a function $f$ on $G$, the left (right) translation of $f$ by $x \in G$ will be denoted by $\left(\ell_{x} f\right)(y)=f(x y)$ (resp. $\left.\left(r_{x} f\right)(y)=f(y x)\right), y \in G$. The standard Lebesgue spaces with respect to the left Haar measure will be denoted by $L^{p}(G), 1 \leq p \leq \infty ; \mathrm{CB}(G)$ will denote the space of all bounded continuous complex-valued functions on $G$ with the supremum norm, and $\operatorname{UC}(G)$ the space of bounded uniformly continuous functions on $G$, i.e. all $f \in \mathrm{CB}(G)$ such that the maps $x \mapsto \ell_{x} f$ and $x \mapsto r_{x} f$ from $G$ into $\operatorname{CB}(G)$ are continuous; $\operatorname{WAP}(G)$ will be the space of continuous weakly almost periodic functions on $G$, i.e. all $f \in \operatorname{CB}(G)$ such that $\left\{\ell_{x} f: x \in G\right\}$ is relatively weakly compact in $\mathrm{CB}(G) ; C_{0}(G)$ the closed subspace of $\operatorname{CB}(G)$ consisting of functions vanishing at infinity; and $C_{\mathrm{c}}(G)$ the dense subspace of $C_{0}(G)$ consisting of functions with compact support.

Let $A(G)$ be all functions $u$ in $C_{0}(G)$ of the form $u(x)=\langle\varrho(x) h, k\rangle$, with $h, k \in L^{2}(G)$ and $\varrho(x) h(y)=h\left(x^{-1} y\right), x, y \in G$, where $\langle$,$\rangle denotes$ the inner product of $L^{2}(G)$. Then $A(G)$ may be identified with the unique predual of $\operatorname{VN}(G)$, the von Neumann algebra on $L^{2}(G)$ generated by $\{\varrho(x)$ : $x \in G\}$. Then, as is well known, $A(G)$ with the predual norm and pointwise multiplication is a commutative Banach algebra with spectrum $G$ (see [7] for details). 
Convolution product of two functions $f$ and $g$ on $G$ is defined by

$$
(f * g)(x)=\int_{G} f(y) g\left(y^{-1} x\right) d \lambda(y),
$$

whenever the integral exists. Then $L^{1}(G)$ is a Banach algebra with convolution product, called the group algebra of $G$. If $G$ is compact, then $L^{p}(G)$, $1 \leq p \leq \infty$, and $C(G)$ are also Banach algebras with convolution product. When $G$ is abelian, $A(G)$ is isometrically and algebraically isomorphic to $L^{1}(\widehat{G})$, where $\widehat{G}$ is the dual group of $G$.

A linear subspace $S(G)$ of the convolution group algebra $L^{1}(G)$ of a locally compact group $G$ is said to be a Segal algebra if it satisfies the following conditions:

(i) $S(G)$ is dense in $L^{1}(G)$;

(ii) $S(G)$ is a Banach space under some norm $\|\cdot\|_{S}$ and $\|f\|_{S} \geq\|f\|_{1}$ for all $f \in S(G)$;

(iii) $S(G)$ is left translation invariant, i.e. $\left\|\ell_{x} f\right\|_{S}=\|f\|_{S}$ for all $x \in G$ and $f \in S(G)$, and the map $x \mapsto \ell_{x} f$ from $G$ into $S(G)$ is continuous.

A Segal algebra $S(G)$ is symmetric if it is right translation invariant, and for each $f \in S(G),\left\|r_{x} f\right\|_{S}=\|f\|_{S}$ for all $x \in G$, and the map $x \mapsto r_{x} f$ from $G$ into $S(G)$ is continuous. Note that the symmetric Segal algebras include all Segal algebras on locally compact abelian groups. Also, every symmetric Segal algebra is a two-sided ideal in $L^{1}(G)$ and has an approximate identity which is self-adjoint with each term having $L^{1}$-norm equal to 1 . The proof of these results and other basic properties of Segal algebras can be found in [22] and [23]. The necessary background on (weak) amenability of Banach algebras can be found in [3].

We recall from [9] that the Lebesgue-Fourier algebra $\mathcal{L} A(G)$ of a locally compact group $G$ is $\mathcal{L} A(G)=L^{1}(G) \cap A(G)$, where

$$
\|f \mid\|=\|f\|_{1}+\|f\|_{A(G)} \quad(f \in \mathcal{L} A(G))
$$

and where the product is the convolution product. As shown in [9], pointwise product also provides a Banach algebra structure on $\mathcal{L} A(G)$.

Let $A$ be a Banach algebra and $X$ be a Banach $A$-module. A derivation $D$ from $A$ into $X$ is approximately inner if there is a net $\left(x_{i}\right) \subset X$ such that

$$
D(a)=\lim _{i \rightarrow \infty}\left[a \cdot x_{i}-x_{i} \cdot a\right] \quad(a \in A) .
$$

The Banach algebra $A$ is approximately amenable if every continuous derivation $D: A \rightarrow X^{*}$ is approximately inner for all Banach $A$-bimodules $X$, and $A$ is approximately weakly amenable if the above holds for $X=A$. The Banach algebra $A$ is weak ${ }^{*}$ approximately weakly amenable if the limit exists in the weak* topology of $A^{*}$ (see [10] for more on these concepts). 
2. Approximate weak amenability of Segal algebras. A locally compact group $G$ is called a $S I N$ group if there is a basis for the neighbourhood of the identity of $e$ consisting of compact sets $U$ such that $x U x^{-1}=U$ for all $x \in G$. Examples of SIN groups include all discrete groups, abelian groups and compact groups. All SIN groups are unimodular.

TheOREM 2.1. Suppose that $S(G)$ is a symmetric Segal algebra on a SIN group $G$. Then:

(i) $S(G)$ is approximately weakly amenable.

(ii) For every continuous derivation $D: S(G) \rightarrow S(G)$ there is a net $\left(\mu_{i}\right) \subset M(G)$ such that $D(f)=\lim _{i \rightarrow \infty}\left[f * \mu_{i}-\mu_{i} * f\right]$.

Proof. (i) Since $G$ is $\operatorname{SIN}, L^{1}(G)$ has a central bounded approximate identity $\left(e_{i}\right)$ such that it is an approximate identity for $S(G)$ (see [20]).

Fix $e_{i}$ and let $n \in S(G)^{*}$. Then for $f \in S(G)$ we have

$$
\left|\left\langle e_{i} \cdot n, f\right\rangle\right|=\left|\left\langle n, f * e_{i}\right\rangle\right| \leq\|n\|_{S(G)^{*}}\left\|f * e_{i}\right\|_{S(G)} \leq\|f\|_{1}\left\|e_{i}\right\|_{S(G)}\|n\|_{S(G)^{*}} .
$$

Hence $e_{i} \cdot n$ is continuous on $\left(S(G),\|\cdot\|_{1}\right)$ and so it has a unique extension to an element of $L^{1}(G)^{*}$ which we denote by $\left(e_{i} \cdot n\right)^{-}$.

Let $D: S(G) \rightarrow S(G)^{*}$ be a continuous derivation. We define $D_{i}:$ $L^{1}(G) \rightarrow S(G)^{*}$ by

$$
D_{i}(f)=D\left(e_{i} * f\right)-D\left(e_{i}\right) \cdot f \quad\left(f \in L^{1}(G)\right) .
$$

Then $D_{i}$ is a derivation (see the proof of Theorem 3.1 in [9]).

Now we define the mapping $\Delta_{i}: L^{1}(G) \rightarrow L^{1}(G)^{*}$ by

$$
\Delta_{i}(f)=\left(e_{i} \cdot D_{i}(f)\right)^{-} \quad\left(f \in L^{1}(G)\right) .
$$

Since $D_{i}$ is a derivation and $\left(e_{i}\right)$ is central, $\Delta_{i}$ is a derivation from $L^{1}(G)$ into $L^{1}(G)^{*}$. Hence by weak amenability of $L^{1}(G)$ (see [5] or [19]), there is $\psi_{i} \in L^{1}(G)^{*}$ such that

$$
\Delta_{i}(f)=f \cdot \psi_{i}-\psi_{i} \cdot f \quad\left(f \in L^{1}(G)\right) .
$$

Let $\varphi_{i}=\psi_{i} \mid S(G)$. Then $\varphi_{i} \in S(G)^{*}$, since the $L^{1}$-norm is dominated by the $S(G)$-norm.

From (1)-(3) for $f \in S(G)$ we have

$$
e_{i} \cdot D\left(e_{i} * f\right)-e_{i} D\left(e_{i}\right) \cdot f=f \cdot \varphi_{i}-\varphi_{i} \cdot f \quad(f \in S(G)),
$$

or

$$
e_{i}^{2} \cdot D(f)=f \cdot \varphi_{i}-\varphi_{i} \cdot f \quad(f \in S(G)) .
$$

Now we note that $\left(e_{i}^{2}\right)$ is an approximate identity for $S(G)$. In fact, for $f \in S(G)$,

$$
\begin{aligned}
\left\|f * e_{i}^{2}-f\right\|_{S(G)} & =\left\|f * e_{i}^{2}-f * e_{i}\right\|_{S(G)}+\left\|f * e_{i}-f\right\|_{S(G)} \\
& \leq\left\|f * e_{i}-f\right\|_{S(G)}\left\|e_{i}\right\|_{L^{1}(G)}+\left\|f * e_{i}-f\right\|_{S(G)} \rightarrow 0 .
\end{aligned}
$$


Hence from (4) we get

$$
D(f)=\text { weak }^{*}-\lim _{i \rightarrow \infty}\left[f \cdot \varphi_{i}-\varphi_{i} \cdot f\right] .
$$

This, in particular, shows that $D\left(e_{i}^{2}\right)=0$, since $\left(e_{i}\right)$ is central. Thus (4) implies that

$$
D\left(e_{i}^{2} * f\right)=f \cdot \varphi_{i}-\varphi_{i} f \quad(f \in S(G))
$$

and so

$$
D(f)=\text { norm- } \lim _{i \rightarrow \infty}\left[f \cdot \varphi_{i}-\varphi_{i} \cdot f\right] \quad(f \in S(G)) .
$$

Hence $S(G)$ is approximately weakly amenable.

(ii) Suppose that $G$ is SIN and $S(G)$ is symmetric and $D: S(G) \rightarrow S(G)$ is a continuous derivation. Let $\left(e_{i}\right)$ be a central approximate identity for $S(G)$, as in the proof of part (i). We define $D_{i}: L^{1}(G) \rightarrow S(G) \subset L^{1}(G)$ by

$$
D_{i}(f)=D\left(e_{i} * f\right)-D\left(e_{i}\right) * f .
$$

By using strong operator topology it can be shown that each $D_{i}$ is a derivation. From [18, Proposition 4.1] or [11, p. 366] there is $\mu_{i} \in M(G)$ such that $D_{i}(f)=f * \mu_{i}-\mu_{i} * f$. In particular, for $f \in S(G)$ we have

$$
e_{i} * D(f)=f * \mu_{i}-\mu_{i} * f \text {. }
$$

Hence

$$
D(f)=\text { norm- } \lim _{i \rightarrow \infty}\left[f * \mu_{i}-\mu_{i} * f\right] \quad(f \in S(G)) .
$$

REMARK 2.2. It was pointed out to us by the referee that an abstract version of Theorem 2.1(i) is also true: Let $A$ be a weakly amenable Banach algebra and let $B$ be an abstract Segal subalgebra of $A$ such that $B$ has a central approximate identity. Then $B$ is approximately weakly amenable. The proof follows exactly that of Theorem 2.1.

REMARK 2.3. Let $G$ be an infinite non-abelian compact group. It is shown in $\left[9\right.$, Remark 3.2] that the convolution Segal algebra $L^{2}(G)$ is not weakly amenable. Thus in general the conclusion of Theorem 2.1 is the best that one can get.

REMARK 2.4. It follows from [8] and Theorem 3.3 in [9] that if $G$ is a totally disconnected amenable locally compact group, then $\mathcal{L} A(G)$ with pointwise multiplication is weakly amenable.

REMARK 2.5. It was shown in [9] that for any locally compact group, $\mathcal{L} A(G)$ with convolution product is amenable if and only if $G$ is discrete and amenable.

3. Derivations from Segal algebras on amenable groups. Suppose that $X$ is a Banach $A$-bimodule. An operator $T: A \rightarrow X$ is a left multiplier if $T(a b)=T(a) \cdot b(a, b \in A)$. Right multipliers are defined similarly. Suppose 
that $T$ is a right multiplier and $S$ is a left multiplier. Then the pair $(S, T)$ is a double centralizer if

$$
a \cdot S(b)=T(a) \cdot b \quad(a, b \in A) .
$$

It is easily verified that when $(S, T)$ is a double centralizer, $D=S-T$ : $A \rightarrow X$ is a derivation.

We recall from [18] that for a Banach algebra $A$, a Banach $A$-bimodule $X$ is neo-unital if $A \cdot X=X \cdot A=X$.

TheOREm 3.1. Let $G$ be an amenable group, and let $S(G)$ be a symmetric Segal algebra on $G$. Suppose that $X$ is a Banach $L^{1}(G)$-bimodule. Then:

(i) For every continuous derivation $D: S(G) \rightarrow X^{*}$ there is a continuous double centralizer $(S, T)$ such that $D=S-T$.

(ii) Every continuous derivation from $S(G)$ into $X$ is approximately inner.

Proof. (i) First we note that if $A$ is a Banach algebra with an approximate identity $\left(e_{i}\right)$, and $X$ is a Banach $A$-bimodule such that the left (or right) action of $A$ on $X$ is trivial, i.e., $a \cdot x=0$ (resp. $x \cdot a=0$ ) for every $a \in A, x \in X$, then every continuous derivation $D: A \rightarrow X$ is approximately inner. In fact if the left action is trivial, then for every $a \in A$,

$$
\begin{aligned}
D(a) & =\lim _{i \rightarrow \infty} D\left(e_{i} * a\right)=\lim _{i \rightarrow \infty}\left[D\left(e_{i}\right) \cdot a+e_{i} \cdot D(a)\right] \\
& =\lim _{i \rightarrow \infty} D\left(e_{i}\right) a=\lim _{i \rightarrow \infty}\left[D\left(e_{i}\right) \cdot a-a \cdot D\left(e_{i}\right)\right] .
\end{aligned}
$$

Now suppose that $X$ is a Banach $L^{1}(G)$-bimodule and $D: S(G) \rightarrow$ $X^{*}$ is a continuous derivation. Let $X_{1}=L^{1}(G) \cdot X \cdot L^{1}(G)$. Then by Cohen's factorization theorem $X_{1}$ is a neo-unital Banach submodule of $X$. By arguments similar to the ones in $\left[18\right.$, p. 15] we can write $X^{*}$ as the direct sum of three submodules one of which is isomorphic to the dual $L^{1}(G)$ module $X_{1}^{*}$, whereas the action of $L^{1}(G)$ on one side of the other two is trivial. Furthermore, the projection of $X^{*}$ on each of these submodules is an $L^{1}(G)$-module morphism. Hence we can write $D=D_{1}+D_{2}+D_{3}$, where $D_{1} \in$ $\mathcal{Z}^{1}\left(S(G), X_{1}^{*}\right)$, whereas $D_{2}$ and $D_{3}$ are approximately inner derivations. So, without loss of generality we can assume that $X$ is a neo-unital Banach $L^{1}(G)$-bimodule.

Let $M u l$ be the Banach space of all continuous left multipliers from $S(G)$ into $X^{*}$. We can make $M u l$ into a Banach $S(G)$-bimodule by defining the right and left actions of $S(G)$ on $M u l$ by

$$
(f \cdot T)(g)=f \cdot T(g), \quad(T \cdot f)(g)=T(f * g) \quad(f, g \in S(G), T \in M u l) .
$$

Suppose $D: S(G) \rightarrow X^{*}$ is a continuous derivation. We can extend $D$ to a continuous derivation $\bar{D}: L^{1}(G) \rightarrow M u l$, where $\bar{D}(f)(g)=D(f * g)-f \cdot D(g)$ 
$\left(f \in L^{1}(G), g \in S(G)\right)$ (see [9, Lemma 4.2]). Hence, from amenability of $L^{1}(G)$, there is a bounded net $\left(T_{j}\right) \subset M u l$ such that

$$
\bar{D}(f)=\lim _{j \rightarrow \infty}\left(f \cdot T_{j}-T_{j} \cdot f\right) \quad\left(f \in L^{1}(G)\right)
$$

([12, Proposition 2.1]). Let $T$ be a cluster point of $\left(T_{j}\right)$ in the weak*-operator topology. Then

$$
\bar{D}(f)=f \cdot T-T \cdot f \quad\left(f \in L^{1}(G)\right) .
$$

Let $\left(e_{i}\right)$ be a bounded approximate identity for $L^{1}(G)$ such that it is also an approximate identity for $S(G)$. Now for $f \in S(G)$ we have

$$
\bar{D}(f)\left(e_{i}\right)=f \cdot T\left(e_{i}\right)-T\left(f * e_{i}\right) .
$$

Hence

$$
D\left(f * e_{i}\right)-f \cdot D\left(e_{i}\right)=f \cdot T\left(e_{i}\right)-T\left(f * e_{i}\right),
$$

or

$$
e_{i} \cdot D(f)=f \cdot T\left(e_{i}\right)-T\left(f * e_{i}\right) .
$$

Therefore

$$
D(f)=\text { weak }^{*}-\lim _{i \rightarrow \infty} f \cdot T\left(e_{i}\right)-T(f) .
$$

Now it is easily seen that $S: f \mapsto$ weak $^{*}-\lim _{i \rightarrow \infty} f \cdot T\left(e_{i}\right)$ is a continuous right multiplier. Furthermore,

$$
\begin{aligned}
S(f) \cdot g & =\text { weak }^{*}-\lim _{i \rightarrow \infty} f \cdot T\left(e_{i}\right) \cdot g=\text { weak }^{*}-\lim _{i \rightarrow \infty} f \cdot T\left(e_{i} * g\right) \\
& =f \cdot T(g) \quad(f, g \in S(G)) .
\end{aligned}
$$

Obviously, $S$ is continuous, since $D$ and $T$ are so. Hence $D=S-T$ for the double centralizer $(S, T)$.

(ii) First suppose that $D \in \mathcal{Z}^{1}\left(S(G), X^{* *}\right)$. Then from equation (1) above we have

$$
D(f)=\text { weak }^{*}-\lim _{i \rightarrow \infty}\left[f \cdot T\left(e_{i}\right)-T\left(e_{i} \cdot f\right)\right]=\text { weak }^{*}-\lim _{i}\left[f \cdot x_{i}^{* *}-x_{i}^{* *} \cdot f\right],
$$

where $x_{i}^{* *}=T\left(e_{i}\right)$.

Now if $D \in \mathcal{Z}^{1}(S(G), X)$, then we have $D \in \mathcal{Z}^{1}\left(S(G), X^{* *}\right)$, through the canonical embedding of $X$ into $X^{* *}$. Hence by the first case there is a net $\left(x_{i}^{* *}\right) \subset X^{* *}$ such that

$$
D(f)=\text { weak }^{*}-\lim _{i \rightarrow \infty}\left[f \cdot x_{i}^{* *}-x_{i}^{* *} \cdot f\right] \quad(f \in S(G)) .
$$

Now it is standard that by using Goldstine's theorem and taking convex combinations we can find a net $\left(x_{i}\right) \subset X$, such that

$$
D(f)=\text { norm- } \lim _{i \rightarrow \infty}\left[f \cdot x_{i}-x_{i} \cdot f\right] \quad(f \in S(G)) .
$$

EXAMPles 3.2. There is a multitude of examples covered by Theorem 3.1. Let $G$ be a unimodular amenable group. Then for any $S(G)$, 
$X$ can be taken to be any of the spaces $L^{p}(G), 1 \leq p<\infty, L^{1}(G) \cap L^{p}(G)$, $1 \leq p<\infty, \operatorname{LUC}(G), \operatorname{RUC}(G), \operatorname{UC}(G), \operatorname{WAP}(G), A(G)$, where the right and left actions of $S(G)$ on $X$ are defined by convolution.

COROLlary 3.3. Let $G$ be an amenable group. Then every symmetric Segal algebra $S(G)$ on $G$ is approximately weakly amenable.

Proof. This follows from part (ii) of Theorem 3.1 with $X=S(G)^{*}$.

REMARK 3.4. The referee has also pointed out that an abstract version of Theorem 3.1 is valid: Let $A$ be an amenable Banach algebra, let $B$ be an abstract Segal subalgebra of $A$ and let $X$ be a Banach $A$-bimodule. Then:

(i') For every continuous derivation $D: B \rightarrow X^{*}$ there is a continuous double centralizer $(S, T)$ such that $D=S-T$.

$\left(i i^{\prime}\right)$ Every continuous derivation from $B$ into $X$ is approximately inner.

(iii') $B$ is approximately weakly amenable.

The proof of these is the same as that of Theorem 3.2.

\section{Derivations and multipliers from Segal algebras on compact} groups. In [9], for a compact group $G$, we characterized all the derivations from the convolution algebra $A(G)$ into $A(G)$. Here, for a compact group $G$, we consider the convolution algebras $C(G), A(G), \mathrm{VN}(G), M(G)$ and $L^{p}(G)$, $1 \leq p \leq \infty$, and attempt to describe continuous derivations from $A$ into $B$, where $A$ and $B$ are chosen from among the above algebras provided that $B$ is naturally a Banach $A$-bimodule. In all the cases, the description of the continuous multipliers from $A$ into $B$ plays an important rôle in identifying the derivations.

In the following theorem $\operatorname{Mul}_{\ell}(A, X)$ stands for the space of all continuous left multipliers from $A$ into $X$, and a subscript $r$ signifies right multipliers. Many of the parts of the theorem are proved in [17, Chapter IX, §35]. Here we provide new proofs for the known ones; our proofs are considerably shorter. We also provide proofs for the previously unknown parts.

TheOREM 4.1. Let $G$ be a compact group. Let $1 \leq p<\infty$ and $q$ be the conjugate exponent to $p$. Then:

(i) $\operatorname{Mul}_{\ell}\left(L^{p}(G), C(G)\right) \cong \operatorname{Mul}_{r}\left(L^{p}(G), C(G)\right) \cong L^{q}(G)$.

(ii) $\operatorname{Mul}_{\ell}\left(L^{p}(G), L^{\infty}(G)\right) \cong \operatorname{Mul}_{r}\left(L^{p}(G), L^{\infty}(G)\right) \cong L^{q}(G)$.

(iii) $\operatorname{Mul}_{\ell}\left(L^{\infty}(G), C(G)\right) \cong \operatorname{Mul}_{r}\left(L^{\infty}(G), C(G)\right) \cong L^{1}(G)$.

(iv) $\operatorname{Mul}_{\ell}(C(G), C(G)) \cong \operatorname{Mul}_{r}(C(G), C(G)) \cong M(G)$.

(v) $M \mathrm{l}_{\ell}\left(C(G), L^{\infty}(G)\right) \cong \operatorname{Mul}_{r}\left(C(G), L^{\infty}(G)\right) \cong M(G)$.

(vi) $\operatorname{Mul}_{\ell}\left(L^{\infty}(G), L^{\infty}(G)\right) \cong \operatorname{Mul}_{r}\left(L^{\infty}(G), L^{\infty}(G)\right) \cong M(G)$.

(vii) $\operatorname{Mul}_{\ell}\left(L^{2}(G), L^{2}(G)\right) \cong \operatorname{Mul}_{r}\left(L^{2}(G), L^{2}(G)\right) \cong \mathrm{VN}(G)$.

(viii) $\operatorname{Mul}_{\ell}(A(G), C(G)) \cong \operatorname{Mul}_{r}(A(G), C(G)) \cong \mathrm{VN}(G)$.

(ix) $\operatorname{Mul}_{\ell}\left(A(G), L^{\infty}(G)\right) \cong \operatorname{Mul}_{r}\left(A(G), L^{\infty}(G)\right) \cong \mathrm{VN}(G)$. 
(x) Let $\Sigma$ be the set of all (equivalence classes of) irreducible unitary representations of $G$, and for $\sigma \in \Sigma$, let $d_{\sigma}$ be the dimension of the Hilbert space which is the range of $\sigma$. If $\sup \left\{d_{\sigma}: \sigma \in \Sigma\right\}<\infty$, then

$$
\operatorname{Mul}_{\ell}(A(G), \mathrm{VN}(G))=M_{u}(A(G), \operatorname{VN}(G))=\mathrm{VN}(G)
$$

and

$$
M u l_{r}(C(G), M(G)) \cong \operatorname{Mul}_{\ell}(C(G), M(G)) \cong \mathrm{VN}(G) .
$$

(xi) In each of the cases above the space of continuous double centralizers coincides with the space of continuous left or right multipliers.

Proof. We prove the results only in the case of left multipliers. Similar proofs can be given in the case of right multipliers.

(i) Suppose that $T \in M u l_{\ell}\left(L^{p}(G), C(G)\right)$. The space $L^{q}(G)$ is a Banach $M(G)$-bimodule when the right and left actions are defined by convolution. It is easily seen that $T^{*}: M(G) \rightarrow L^{q}(G)$ is a left multiplier, and so, as $M(G)$ is unital, there is $g \in L^{q}(G)$ such that $T^{*}(\mu)=g * \mu(\mu \in M(G))$. Now for $f \in L^{p}(G)$ and $\mu \in M(G)$ we have

$$
\langle T(f), \mu\rangle=\left\langle f, T^{*}(\mu)\right\rangle=\langle f, g * \mu\rangle=\langle\check{g} * f, \mu\rangle,
$$

where $\check{g}(x)=g\left(x^{-1}\right)(x \in G)$. Hence

$$
T(f)=\check{g} * f \quad\left(f \in L^{p}(G)\right) .
$$

(ii) Let $T \in M u l_{\ell}\left(L^{p}(G), L^{\infty}(G)\right)$, and let $\left(e_{i}\right)$ be an approximate identity for $L^{p}(G)$. Then

$$
T(f)=\lim _{i \rightarrow \infty} T\left(e_{i} * f\right)=\lim _{i \rightarrow \infty} T\left(e_{i}\right) * f \in C(G) .
$$

Hence the result follows from (i).

(iii) Let $T \in \operatorname{Mul}_{\ell}\left(L^{\infty}(G), C(G)\right)$. The Banach space $L^{1}(G)^{* *}$ is a Banach $M(G)$-bimodule since $L^{1}(G)$ is a Banach $M(G)$-bimodule with actions defined by convolution. The operator $T^{*}: M(G) \rightarrow L^{1}(G)^{* *}$ is a left multiplier. In particular $T^{*} \mid L^{1}(G): L^{1}(G) \rightarrow L^{1}(G)^{* *}$ is a left multiplier. Let $\left(e_{i}\right)$ be a bounded approximate identity for $L^{1}(G)$. For every $f \in L^{1}(G)$ we have $T(f)=$ norm- $\lim _{i \rightarrow \infty} T\left(e_{i}\right) * f \in L^{1}(G)$, since $L^{1}(G)$ is an ideal in $L^{1}(G)^{* *}$. Hence $T^{*}$ restricts to a left multiplier from $L^{1}(G)$ into $L^{1}(G)$. Thus by Wendel's characterization of multipliers of $L^{1}(G)$, there is a measure $\mu \in M(G)$ such that $T^{*}(f)=\mu * f$ for every $f \in L^{1}(G)$. Now for $g \in L^{\infty}(G)$ and $f \in L^{1}(G)$ we have

$$
\langle T(g), f\rangle=\left\langle g, T^{*} f\right\rangle=\langle g, \mu * f\rangle=\langle\check{\mu} * g, f\rangle,
$$

where $\check{\mu}$ is the measure defined by

$$
\langle\check{\mu}, \varphi\rangle=\langle\mu, \check{\varphi}\rangle \quad(\varphi \in C(G)) .
$$

Hence $T(g)=\check{\mu} * g\left(g \in L^{\infty}(G)\right)$. Since $T$ maps into $C(G)$, we conclude from [16, V.19.27] that $\mu \in L^{1}(G)$. 
(iv) Similar to (i).

(v) Let $T \in \operatorname{Mul}_{\ell}\left(C(G), L^{\infty}(G)\right)$. Let $\left(e_{i}\right)$ be an approximate identity for $C(G)$. Then for $f \in C(G), T(f)=\lim _{i \rightarrow \infty} T\left(e_{i}\right) * f \in C(G)$, and the result follows from (iv).

(vi) Let $T \in M u l_{\ell}\left(L^{\infty}(G), L^{\infty}(G)\right)$. By (v) there is a measure $\mu \in M(G)$ such that $T(f)=\mu * f$ for all $f \in C(G)$. The relation $T(f * g)=T(f) * g$, which is valid for $f, g \in L^{\infty}(G)$, can be extended to hold for all $g \in L^{1}(G)$, by continuity and since $L^{\infty}(G)$ is dense in $L^{1}(G)$.

Now for $f \in L^{\infty}(G)$ and $g_{1}, g_{2} \in L^{1}(G)$,

$$
\begin{aligned}
\left\langle T(f), g_{1} * g_{2}\right\rangle & =\left\langle T(f) * \check{g}_{2}, f_{1}\right\rangle=\left\langle T\left(f * \check{g}_{2}\right), g_{1}\right\rangle=\left\langle\mu * f * \check{g}_{2}, g_{1}\right\rangle \\
& =\left\langle\mu * f, g_{1} * g_{2}\right\rangle,
\end{aligned}
$$

and we have the result since $L^{1}(G)$ factorizes.

(vii) For $n \in \mathrm{VN}(G)$, let $\lambda_{n}$ be an operator on $L^{2}(G)$, given by the duality $\left\langle\lambda_{n}(f), g\right\rangle_{2}=\langle n, f * g\rangle$, where the last number stands for the value of $n \in A(G)^{*}$ at $f * g \in A(G)$. It is readily seen that $\lambda_{n}$ is a left multiplier.

For the converse suppose that $T \in M u l_{\ell}\left(L^{2}(G), L^{2}(G)\right)$. We define an operator $\widetilde{T}: A(G) \rightarrow A(G)$ by

$$
\widetilde{T}(h)=T(f) * \check{g} \quad \text { if } h=f * \check{g}
$$

for some $f, g \in L^{2}(G)$. We notice that $\widetilde{T}$ is well defined since the right hand side can be written as $\lim _{i \rightarrow \infty} T\left(e_{i}\right) * f * \check{g}=\lim _{i \rightarrow \infty} T\left(e_{i}\right) * h$, where $\left(e_{i}\right)$ is an approximate identity for $L^{2}(G)$. Now from [9, Theorem 5.2] there is $n \in \mathrm{VN}(G)$ such that $\widetilde{T}(h)=n \cdot h(h \in A(G))$. Hence from equation (1) we have

$$
T(f) * \check{g}=n \cdot(f * \check{g}) .
$$

By letting $g=\check{e}_{i}$ and taking norm limits, we obtain $T(f)=n \cdot f$.

(viii) Similar to (iii).

(ix) Let $T \in \operatorname{Mul}_{\ell}\left(A(G), L^{\infty}(G)\right)$. By using an approximate identity of $A(G)$ we see that $T$ maps into $C(G)$. Hence the result follows from (viii).

(x) It is shown in [17] that under the stated condition, $\operatorname{Mul}_{\ell}\left(L^{2}(G)\right.$, $\mathrm{VN}(G))=\mathrm{VN}(G)$. Suppose $T \in M u l_{\ell}(A(G), \mathrm{VN}(G))$. Define an operator $\widetilde{T}: L^{2}(G) \rightarrow \operatorname{Mul}\left(L^{2}(G), \mathrm{VN}(G)\right)=\mathrm{VN}(G)$ by

$$
\widetilde{T}(f)(g)=T(f * g) \quad\left(g \in L^{2}(G)\right) .
$$

There is an $n \in \mathrm{VN}(G)$ such that $\widetilde{T}(f)=n \cdot f$. Hence $T(f * g)=n \cdot(f * g)$, for $f, g \in L^{2}(G)$ and we have the result, since $A(G)=L^{2}(G) * L^{2}(G)$.

To prove the second part, first we notice that to every $n \in \operatorname{VN}(G)$ and $f \in C(G)$, we can associate an element $n \cdot f \in M(G)$, specified by $\langle n \cdot f, g\rangle=\langle n, f * g\rangle$, where the last pairing is $(\mathrm{VN}(G), A(G))$ dual pairing. 
We notice that since

$$
|\langle n, f * g\rangle| \leq\|n\|\|f * g\| \leq\|n\|\|f\|_{2}\|g\|_{2} \leq\|n\|\|f\|_{C(G)}\|g\|_{C(G)},
$$

the element $n \cdot f$ as defined above is a continuous functional on $C(G)$. Hence $f \mapsto n \cdot f$ is a multiplier from $C(G)$ into $M(G)$.

For the converse, suppose that $T \in \operatorname{Mul}_{\ell}(C(G), M(G))$. Then $T \mid A(G)$ is a multiplier from $A(G)$ to $M(G) \hookrightarrow \mathrm{VN}(G)$. By the first part there is $n \in \mathrm{VN}(G)$ such that $T(f)=n \cdot f$ for all $f \in A(G)$, and since $A(G)$ is dense in $C(G)$, this holds for all $f \in C(G)$.

(xi) We prove this only in the case of double centralizers from $L^{p}(G)$ into $C(G)$. The proofs of the other cases follow similar lines. So let $(S, T)$ be a continuous double centralizer from $C(G)$ into $L^{p}(G)$. Then by (1) there are $f_{1}, f_{2} \in L^{q}(G)$ such that $g_{1} * f_{1} * g_{2}=g_{1} * f_{2} * g_{2}\left(g_{1}, g_{2} \in C(G)\right)$. By using an approximate identity we obtain $f_{1}=f_{2}$.

THEOREM 4.2. Let $G$ be a compact group, $1 \leq p<\infty$ and $q$ be the conjugate exponent to $p$. Then:

(i) $D \in \mathcal{Z}^{1}\left(L^{p}(G), L^{\infty}(G)\right)$ if and only if there is $g \in L^{q}(G)$ such that $D(f)=f * g-g * f\left(f \in L^{p}(G)\right)$.

(ii) $D \in \mathcal{Z}^{1}\left(L^{p}(G), C(G)\right)$ if and only if there is $g \in L^{q}(G)$ such that $D(f)=f * g-g * f\left(f \in L^{p}(G)\right)$.

(iii) $D \in \mathcal{Z}^{1}\left(L^{\infty}(G), C(G)\right)$ if and only if there is $g \in L^{1}(G)$ such that $D(f)=f * g-g * f\left(f \in L^{\infty}(G)\right)$.

(iv) $D \in \mathcal{Z}^{1}(C(G), C(G))$ if and only if there is $\mu \in M(G)$ such that $D(f)=f * \mu-\mu * f(f \in C(G))$.

(v) $D \in \mathcal{Z}^{1}\left(C(G), L^{\infty}(G)\right)$ if and only if there is $\mu \in M(G)$ such that $D(f)=f * \mu-\mu * f(f \in C(G))$.

(vi) $D \in \mathcal{Z}^{1}\left(L^{\infty}(G), L^{\infty}(G)\right)$ if and only if there is $\mu \in M(G)$ such that $D(f)=f * \mu-\mu * f\left(f \in L^{\infty}(G)\right)$.

(vii) $D \in \mathcal{Z}^{1}\left(L^{2}(G), L^{2}(G)\right)$ if and only if there is $n \in \mathrm{VN}(G)$ such that $D(f)=f \cdot n-n \cdot f\left(f \in L^{2}(G)\right)$.

(viii) $D \in \mathcal{Z}^{1}(A(G), C(G))$ if and only if there is $n \in \mathrm{VN}(G)$ such that $D(f)=f \cdot n-n \cdot f(f \in A(G))$.

(ix) $D \in \mathcal{Z}^{1}\left(A(G), L^{\infty}(G)\right)$ if and only if there is $n \in \mathrm{VN}(G)$ such that $D(f)=f \cdot n-n \cdot f(f \in A(G))$.

(x) Furthermore, if $\sup \left\{d_{\sigma}: \sigma \in \Sigma\right\}<\infty$, then $D \in \mathcal{Z}^{1}(A(G), \operatorname{VN}(G))$ if and only if there is $n \in \mathrm{VN}(G)$ such that $D(f)=f \cdot n-n \cdot f$ $(f \in A(G))$, and $D \in \mathcal{Z}^{1}(C(G), M(G))$ if and only if there is $n \in \mathrm{VN}(G)$ such that $D(f)=f \cdot n-n \cdot f(f \in C(G))$.

Proof. Parts (i), (v), (vi), (vii), (ix) and (x) follow from part (i) of Theorem 3.1 and from Theorem 4.1. Then we have (ii), (iv), (vii) since (i) $\Rightarrow($ ii), (v) $\Rightarrow$ (iv) and (vii) $\Rightarrow$ (viii). Now to prove (vi), let $D \in \mathcal{Z}^{1}\left(L^{\infty}(G), L^{\infty}(G)\right)$. 
By (v) there is $\mu \in M(G)$ such that for every $f \in C(G), D(f)=f * \mu-\mu * f$. To prove (iii), let $f \in L^{\infty}(G), g_{1}, g_{2} \in C(G)$. Then

$$
\begin{aligned}
\left\langle D(f), g_{1} * g_{2}\right\rangle & =\left\langle D(f) \cdot g_{1}, g_{2}\right\rangle=\left\langle\check{g}_{1} * D(f), g_{2}\right\rangle \\
& =\left\langle D\left(\check{g}_{1} * f\right)-D\left(\check{g}_{1}\right) * f, g_{2}\right\rangle \\
& =\left\langle\check{g}_{1} * f * \mu-\mu * \check{g}_{1} * f-\check{g}_{1} * \mu * f+\mu * \check{g}_{1} * f, g_{2}\right\rangle \\
& =\left\langle f * \mu-\mu * f, g_{1} * g_{2}\right\rangle .
\end{aligned}
$$

Hence $D(f)=f * \mu-\mu * f$, since $C(G) * C(G)$ is dense in $L^{1}(G)$.

5. Arens regularity of $\mathcal{L} A(G)$. The second dual space $A^{* *}$ of a Banach algebra $A$ can be equipped with two Banach algebra products, called first and second Arens products, each of which extends the original product of $A$ as canonically embedded in $A^{* *}$. The Banach algebra $A$ is Arens regular if the two Arens products on $A^{* *}$ coincide ([6], [3]). A condition equivalent to Arens regularity of $A$ due to J. S. Pym is that for every $f \in A^{*}$, the operator $a \mapsto a \cdot f: A \rightarrow A^{*}$ is weakly compact [6]. Here $a \cdot f \in A^{*}$ is defined by

$$
\langle a \cdot f, b\rangle=\langle f, b a\rangle \quad(b \in A)
$$

(see $[6])$.

TheOREm 5.1. Suppose that $S(G)$ is a symmetric Segal subalgebra of $L^{1}(G)$. Then:

(i) Arens regularity of $S(G)$ is sufficient but not necessary for $G$ to be compact.

(ii) Let $G$ be unimodular. Then $\mathcal{L} A(G)$ is Arens regular if and only if $G$ is compact.

Proof. (i) We denote the norm on $S(G)$ by $\|\cdot\|_{S}$. Take $f \in \mathrm{UC}(G)$. Since for every $g \in S(G)$,

$$
|\langle f, g\rangle|=\left|\int_{G} f(x) g(x) d x\right| \leq\|f\|_{\infty}\|g\|_{1} \leq\|f\|_{\infty}\|g\|_{S},
$$

we can view $f$ as an element of $S(G)^{*}$, through integration. Let $\left(e_{i}\right)$ be a bounded approximate identity for $L^{1}(G)$ such that it is also an approximate identity for $S(G)$. Fix $e_{i}$. For $g \in L^{1}(G)$ we have $\left\|g * e_{i}\right\|_{S} \leq\|g\|_{1}\left\|e_{i}\right\|_{S}$ and so the set $\left\{g * e_{i}:\|g\|_{1} \leq 1\right\}$ is bounded in $S(G)$. Hence by our assumption on Arens regularity of $S(G)$, the set $\left\{\left(g * e_{i}\right) \cdot f:\|g\|_{1} \leq 1\right\}$ has weakly compact closure in $S(G)^{*}$. The operator $T_{i}: f \mapsto f \cdot e_{i}$ from $S(G)^{*}$ into $L^{1}(G)^{*}$ is continuous, since for every $g \in L^{1}(G)$,

$$
\left|\left\langle f \cdot e_{i}, g\right\rangle\right|=\left|\left\langle f, e_{i} * g\right\rangle\right| \leq\|f\|\left\|e_{i} * g\right\|_{S} \leq\|f\|\left\|e_{i}\right\|_{S}\|g\|_{1} .
$$

Hence the set $\left\{\left(g * e_{i}\right) \cdot f \cdot e_{i}:\|g\|_{1} \leq 1\right\}$ has weakly compact closure in $L^{\infty}(G)$. This means that $e_{i} \cdot f \cdot e_{i}$ is a weakly almost periodic function on $G$. 
We also have $f=\lim _{i \rightarrow \infty} e_{i} \cdot f \cdot e_{i}$, since $\mathrm{UC}(G)=L^{1}(G) * \mathrm{UC}(G) * L^{1}(G)$. Thus $f \in \operatorname{WAP}(G)$. Hence $\operatorname{WAP}(G)=\operatorname{UC}(G)$ and so $G$ is compact [13].

To show that the converse is not true, we let $G$ be an infinite compact group and we consider $S(G)=L^{1}(G)$, which is not Arens regular [25].

(ii) If $G$ is unimodular, $\mathcal{L} A(G)$ is a symmetric Segal algebra. Hence the result follows from part (i) and $[9$, Theorem 6.2].

If $G$ is a locally compact group, let $\operatorname{UC}(\widehat{G})$ denote the closed linear span of $\{u \cdot T: u \in A(G)$ and $T \in \operatorname{VN}(G)\}$. When $G$ is amenable, then $\mathrm{UC}(\widehat{G})=A(G) \cdot \operatorname{VN}(G)$ by Cohen's factorization theorem as shown by Granirer [13]. When $G$ is abelian, $\operatorname{UC}(\widehat{G})$ is precisely the space of bounded uniformly continuous functions on the dual group $\widehat{G}$. Let $\operatorname{WAP}(\widehat{G})$ denote the set of all $T \in \operatorname{VN}(G)$ such that $\{u \cdot T: u \in A(G),\|u\| \leq 1\}$ is relatively compact in the weak topology of $\operatorname{VN}(G)$. When $G$ is abelian, $\operatorname{WAP}(\widehat{G})$ is the space of continuous weakly almost periodic functions on $\widehat{G}$.

THeOREM 5.2. Let $G$ be a locally compact group. Then $\mathcal{L} A(G)$ with pointwise multiplication is Arens regular if and only if $G$ is discrete.

Proof. The "if" part is proved in [9, Proposition 6.1]. For the converse, we will show that if $\mathcal{L} A(G)$ is Arens regular, $\operatorname{UC}(\widehat{G})=\operatorname{WAP}(\widehat{G})$. Then $G$ must be discrete by using [13] and [14]. Since $\mathcal{L} A(G)$ is norm dense in $A(G)$, it suffices to show that if $v \in \mathcal{L} A(G), T \in \operatorname{VN}(G)$, then $S=v \cdot T \in \operatorname{WAP}(\widehat{G})$. We may view $S$ as an element of $\mathcal{L} A(G)^{*}$.

Let $\||\cdot| \mid$ denote the norm in $\mathcal{L} A(G)$. Then since

$$
\||u v\||\leq\|u\||\| v\|\|,
$$

the set $\{u v:\|u\| \leq 1, u \in A(G)\}$ is a norm bounded subset of $\mathcal{L} A(G)$. So $\{u \cdot S:\|u\| \leq 1, u \in A(G)\}$ is relatively weakly compact in $\mathcal{L} A(G)^{*}$ by Arens regularity of $\mathcal{L} A(G)$. Consider now the map $\Pi: \mathcal{L} A(G)^{*} \rightarrow \operatorname{VN}(G)$ defined by $\Pi(T)=v \cdot T$. Then for $u \in A(G)$, we have

$$
|\langle v \cdot T, u\rangle|=|\langle T, v u\rangle| \leq\|T\|_{\mathcal{L} A(G)^{*}}\left|\left\|v u \left|\|\leq\| T\left\|_{\mathcal{L} A(G)^{*}} \mid\right\| v\|\|\|u\|_{A(G)}\right.\right.\right.
$$

(since $\mathcal{L} A(G)$ is an ideal in $A(G)$ ). Hence $\Pi$ is continuous and so the set $\{\Pi(u \cdot S): u \in A(G),\|u\| \leq 1\}=\{u \cdot(v \cdot T): u \in A(G):\|u\| \leq 1\}$ is relatively weakly compact in $\operatorname{VN}(G)$. Thus $S \in \operatorname{WAP}(\widehat{G})$ as required.

After the completion of our paper, Professor E. E. Granirer kindly sent us a preprint of his very interesting work [15] where a proof of the "only if" direction of our Theorem 5.2 is given. However his proof is for a much more general situation and it is in fact quite different from ours.

REMARK 5.3. When $G$ is a compact group each of the Segal algebras $(C(G), *)$ and $\left(L^{\infty}(G), *\right)$ is Arens regular. This is due to the fact that 
every bounded operator from a $C^{*}$-algebra into its dual space is weakly compact [1].

6. $\mathcal{L} A(G)$ as an ideal in its second dual space. Suppose that $A$ is a Banach algebra and the second dual space $A^{* *}$ of $A$ is equipped with the first or the second Arens product. It is well known that $A$ is an ideal in $A^{* *}$ if and only if, for every $a \in A$, the operators $\varrho_{a}: b \mapsto a b$ and $\lambda_{a}: b \mapsto b a$ are weakly compact [6].

Proposition 6.1. Let $S(G)$ be a symmetric Segal subalgebra of $L^{1}(G)$. Then the following are equivalent:

(i) $G$ is compact;

(ii) for every $f \in S(G), \lambda_{f}: g \mapsto f * g(g \in S(G))$ is compact;

(iii) for every $f \in S(G), \lambda_{f}: g \mapsto f * g(g \in S(G))$ is weakly compact;

(iv) for some non-zero $f \in S(G), \lambda_{f}: g \mapsto f * g(g \in S(G))$ is compact;

(v) for some non-zero $f \in S(G), \lambda_{f}: g \mapsto f * g(g \in S(G))$ is weakly compact.

Proof. (i) $\Rightarrow$ (ii). Let $G$ be compact. It is known ([2]) that for every $f \in$ $L^{1}(G), \lambda: g \mapsto f * g$ is a compact operator on $L^{1}(G)$. Now let $f \in S(G)$. Then $f=g * h$ for some $h \in L^{1}(G)$ and $g \in S(G)$. Let $\left(k_{n}\right)$ be a bounded sequence in $S(G)$. Then $\left(k_{n}\right)$ is bounded in $L^{1}(G)$. Hence for a subsequence $\left(k_{n_{i}}\right)$ of $\left(k_{n}\right)$, the sequence $\left(h * k_{n_{i}}\right)$ converges in $L^{1}(G)$, and so $\left(g * h * k_{n_{i}}\right)$ converges in $S(G)$.

The implications (ii) $\Rightarrow$ (iii) and (iii) $\Rightarrow$ (iv) $\Rightarrow($ v) are clear.

$(\mathrm{v}) \Rightarrow(\mathrm{i})$. Let $f \in S(G), f \neq 0$, and suppose that $\lambda_{f}: g \mapsto f * g$ is a weakly compact operator on $S(G)$. Let $\left(k_{n}\right)$ be a bounded sequence in $L^{1}(G)$. Let $h \in S(G)$ be such that $f * h \neq 0$ (such $h$ exists since $S(G)$ contains a bounded approximate identity of $\left.L^{1}(G)\right)$. Then $\left(h * k_{n}\right)$ is a bounded sequence in $S(G)$ and so by weak compactness of $\lambda_{f}$, there is a subsequence $\left(h * k_{n_{i}}\right)$ of $\left(h * k_{n}\right)$ such that $\left(f * h * k_{n_{i}}\right)$ converges weakly in $S(G)$. Choose $m \in L^{1}(G)$ with $m * f * h \neq 0$. Then the sequence $m * f * h * k_{n_{i}}$ converges weakly in $L^{1}(G)$. This shows that for the non-zero element $p=m * f * h$ of $L^{1}(G), \lambda_{p}: L^{1}(G) \rightarrow L^{1}(G)$ is weakly compact, and this in turn forces $G$ to be compact $[2]$.

Corollary 6.2. Let $G$ be a unimodular group. Then the algebra $\mathcal{L} A(G)$ is an ideal in its second dual if and only if $G$ is compact.

Proof. When $G$ is unimodular, $\mathcal{L} A(G)$ is a symmetric Segal subalgebra of $L^{1}(G)$.

We now let $\mathcal{L} A(G)$ have a pointwise product and determine when it is an ideal in its second dual. First we need the following: 
We recall that a scalar-valued function $f$ on $G$ is positive-definite if for any finite number of complex numbers $c_{1}, \ldots, c_{n}$ and elements $x_{1}, \ldots, x_{n}$ of $G$ we have $\sum_{i, j} c_{i} \bar{c}_{j} f\left(x_{i} x_{j}^{-1}\right) \geq 0$. The Fourier-Stieltjes algebra $B(G)$ of $G$ is the linear span of all continuous positive-definite functions on $G$, with the pointwise multiplication [7]. Let

$$
P_{1}(G)=\left\{\varphi \in B(G): \varphi(e)=1=\|\varphi\|_{B(G)}\right\} .
$$

That is, $P_{1}(G)$ consists of all positive-definite functions $\varphi$ on $G$ with $\varphi(e)$ $=1$. For every $f \in \mathcal{L} A(G)$ we let

$$
K_{f}:=\left\{v f: v \in P_{1}(G) \cap A(G)\right\} .
$$

We note that $K_{f} \subset \mathcal{L} A(G)$, since $\|v f\|_{A(G)}=\|f\|_{A(G)}$ and $\|v f\|_{1} \leq\|f\|_{1}$.

Lemma 6.3. Suppose that $f \in \mathcal{L} A(G), f$ is positive-definite and $f(e)=1$ $(\|f\|=1)$. If $K_{f}$ is relatively weakly compact in $\mathcal{L} A(G)$, then $G$ is discrete.

Proof. Since the inclusion map $\iota: \mathcal{L} A(G) \rightarrow A(G)$ is continuous, it follows that $K_{f}$ is a relatively weakly compact subset of $A(G)$. Now $K_{f}$ is convex and so $\bar{K}_{f}$ is a weakly compact convex subset of $A(G)$ contained in $P_{1}(G) \cap A(G)$. For every $v \in P_{1}(G)$, let $T_{v}$ be a mapping on $\bar{K}_{f}$ defined by $T_{v}(w)=v w\left(w \in \bar{K}_{f}\right)$, and let $S=\left\{T_{v}: v \in P_{1}(G)\right\}$. Then $S$ is a commuting family of continuous affine mappings on $\bar{K}_{f}$. So by the MarkovKakutani fixed point theorem there is $w_{0} \in \bar{K}_{f}$ such that $v w_{0}=w_{0}$ for every $v \in P_{1}(G)$. Hence $w_{0}$ is a topological invariant mean on $\operatorname{VN}(G)$ and $w_{0} \in A(G)$. Then by a result of [24], $G$ must be discrete.

THEOREM 6.4. Let $G$ be a locally compact group and suppose that $\mathcal{L} A(G)$ is equipped with the pointwise product. Then $\mathcal{L} A(G)$ is an ideal in $\mathcal{L} A(G)^{* *}$ if and only if $G$ is discrete.

Proof. If $G$ is discrete, then $\mathcal{L} A(G)=\ell^{1}(G)$ with pointwise product and this is a compact algebra. For the converse, suppose that $\mathcal{L} A(G)$ is an ideal in $\mathcal{L} A(G)^{* *}$. Then for every $f \in \mathcal{L} A(G)$ the set $Q_{f}=\{w f:\|w\| \leq 1$, $w \in \mathcal{L} A(G)\}$ is relatively compact in the weak topology of $\mathcal{L} A(G)$. Now, $\mathcal{L} A(G)$ contains all the functions in $A(G)$ with compact support.

Take $h \in C_{\mathrm{c}}(G)$ with $\|h\|_{2}=1$, and set $f(x)=\langle\varrho(x) h, h\rangle(x \in G)$. Then $f \in A(G) \cap C_{\mathrm{c}}(G), f$ is positive-definite, $f(e)=1$, and $Q_{f}$ is relatively weakly compact in $\mathcal{L} A(G)$. Now, for $K_{f}$ as defined before Lemma 6.3 we have

$$
K_{f^{2}}=\left\{v f^{2}: v \in P_{1}(G) \cap A(G)\right\}=\left\{(v f) f: v \in P_{1}(G) \cap A(G)\right\} .
$$

For $v f$ as above we have

$$
\|v f\|_{A(G)}=(v f)(e)=f(e)=\|f\|_{A(G)},
$$


and

$$
\|v f\|_{1}=\int_{G}|v(t)| f(t) d t \leq\|f\|_{1} .
$$

Therefore $K_{f^{2}} \subseteq\left(\|f\|_{A(G)}+\|f\|_{1}\right) Q_{f}$. Hence $K_{f^{2}}$ is relatively weakly compact in $\mathcal{L} A(G)$, and so $G$ is discrete by Lemma 6.3 .

REMARK 6.5. The proof of Lemma 6.3 shows that if there exists an $f \in A(G), f \geq 0$ and positive-definite, with $f(e)=1$, such that $g \mapsto f \cdot g$ is a weakly compact operator from $A(G)$ into $A(G)$, then $G$ is discrete.

Open Questions. 1. Is Theorem 1.1 true without $G$ being a SIN group?

2 . Is every symmetric Segal algebra on an amenable group approximately amenable?

3. Suppose that there exists an $f \in A(G), f \neq 0$, such that $g \mapsto f g$ is a weakly compact operator from $A(G)$ into $A(G)$. Is $G$ discrete?

\section{References}

[1] C. A. Akemann, The dual space of an operator algebra, Trans. Amer. Math. Soc. 126 (1967), 286-302.

[2] - Some mapping properties of the group algebras of a compact group, Pacific J. Math. 22 (1967), 1-8.

[3] H. G. Dales, Banach Algebras and Automatic Continuity, Clarendon Press, Oxford, 2000 .

[4] H. G. Dales and S. S. Pandey, Weak amenability of Segal algebras, Proc. Amer. Math. Soc. 128 (2000), 1419-1425.

[5] M. Despić and F. Ghahramani, Weak amenability of group algebras of locally compact groups, Canad. Math. Bull. 37 (1994), 165-167.

[6] J. Duncan and S. A. R. Hosseiniun, The second dual of a Banach algebra, Proc. Roy. Soc. Edinburgh Sect. A 84 (1979), 309-325.

[7] P. Eymard, L'algèbre de Fourier d'un groupe localement compact, Bull. Soc. Math. France 92 (1964), 181-236.

[8] B. E. Forrest, Fourier analysis on coset spaces, Rocky Mountain J. Math. 28 (1998), 173-190.

[9] F. Ghahramani and A. T.-M. Lau, Weak amenability of certain classes of Banach algebras without bounded approximate identities, Math. Proc. Cambridge Philos. Soc. 133 (2002), 357-371.

[10] F. Ghahramani and R. J. Loy, Generalized notions of amenability, J. Funct. Anal. 208 (2004), 229-260.

[11] F. Ghahramani, V. Runde and G. A. Willis, Derivations on group algebras, Proc. London Math. Soc. (3) 80 (2000), 360-390.

[12] F. Gourdeau, Amenability and the second dual of a Banach algebra, Studia Math. 125 (1997), 75-81.

[13] E. E. Granirer, Weakly almost periodic and uniformly continuous functionals on the Fourier algebra of any locally compact group, Trans. Amer. Math. Soc. 189 (1974), 371-382.

[14] - Density theorems for some linear subspaces and some $C^{*}$-subalgebras of $\operatorname{VN}(G)$, in: Sympos. Math. 22, Academic Press, London, 1977, 61-70. 
[15] E. E. Granirer, The Fourier-Herz-Lebesgue Banach algebras, preprint.

[16] E. Hewitt and K. A. Ross, Abstract Harmonic Analysis, Vol. I, 2nd ed., Springer, Berlin, 1979.

[17] —, -, Abstract Harmonic Analysis, Vol. II, Springer, Berlin, 1970.

[18] B. E. Johnson, Cohomology in Banach algebras, Mem. Amer. Math. Soc. 127 (1972).

[19] —, Weak amenability of group algebras, Bull. London Math. Soc. 23 (1991), 281-284.

[20] E. Kotzmann and H. Rindler, Segal algebras on non-abelian groups, Trans. Amer. Math. Soc. 237 (1978), 271-281.

[21] A. T.-M. Lau, Uniformly continuous functionals on the Fourier algebra of any locally compact group, ibid. 251 (1979), 39-59.

[22] H. Reiter, $L^{1}$-algebras and Segal Algebras, Lecture Notes in Math. 231, Springer, Berlin, 1971.

[23] H. Reiter and J. D. Stegeman, Classical Harmonic Analysis and Locally Compact Groups, Clarendon Press, Oxford, 2000.

[24] P. F. Renaud, Invariant means on a class of von Neumann algebras, Trans. Amer. Math. Soc. 170 (1972), 285-291.

[25] N. J. Young, The irregularity of multiplication in group algebras, Quart. J. Math. Oxford Ser. (2) 24 (1973), 59-62.

Department of Mathematics

University of Manitoba

Winnipeg, Canada R3T 2N2

E-mail: fereidou@cc.umanitoba.ca
Department of Mathematical and Statistical Sciences University of Alberta

Edmonton, Canada T6G 2G1

E-mail: tlau@math.ualberta.ca

Received October 13, 2004

Revised version January 21, 2005 